\title{
Pharmacotherapeutic Problems and Pharmacist Interventions in a Medical Intensive Care Unit
}

\author{
Tae Yun Park, M.D., Sang-Min Lee, M.D., Ph.D., Sung Eun Kim, RPh", Ka-Eun Yoo, RPh", Go Wun Choi, RPh \\ Yun Hee Jo, RPh", Yoonsook Cho, RPh", Hyeon Joo Hahn, RPh", Jinwoo Lee, M.D., and A Jeong Kim, RPh \\ Department of Internal Medicine, *Department of Pharmacy, Seoul National University Hospital, Seoul, Korea
}

Background: Interest in pharmacist participation in the multidisciplinary intensive care team is increasing. However, studies examining pharmacist interventions in the medical intensive care unit (MICU) are limited in Korea. The aim of this study was to describe the current status of pharmacist interventions and to identify common pharmacologic problems requiring pharmacist intervention in the MICU.

Methods: Between September 2013 and August 2014, a retrospective, observational study was conducted in the 22-bed MICU at a university hospital. Data were obtained from two trained pharmacists who participated in MICU rounds three times a week. In addition to patient characteristics, data on the cause, type, related drug, and acceptance rate of interventions were collected.

Results: In 340 patients, a total of 1211 pharmacologic interventions were performed. The majority of pharmacologic interventions were suggested by pharmacists at multidisciplinary rounds in the MICU. The most common pharmacologic interventions were adjustment of dosage and administration $(n=328,26.0 \%)$, followed by parenteral/enteral nutritional support $(n=228,18.1 \%)$, the provision of drug information $(n=228,18.1 \%)$, and advice regarding pharmacokinetics $(n=118,9.3 \%)$. Antimicrobial agents $(n=516,42.6 \%)$ were the most frequent type of drug associated with pharmacist interventions. The acceptance rate of interventions was $84.1 \%$ with most accepted by physicians within 24 hours ( $n=602,92.8 \%$ ).

Conclusions: Medication and nutritional problems are frequently encountered pharmacotherapeutic problems in the MICU. Pharmacist interventions play an important role in the management of these problems.

Key Words: intensive care unit; intervention; pharmacist.

\section{Introduction}

Interest in the participation of pharmacists in multidisciplinary intensive care teams is increasing. It has been shown previously that pharmacist interventions have beneficial effects in critically ill patients, including in terms of reducing the incidence of medication errors and adverse drug events.[1-4] Despite these beneficial effects, independent clinical pharmacy services in the intensive care unit (ICU) were adopted only recently in Korea.[5]

Previously, studies of pharmacist interventions in surgical intensive care units (SICUs) were performed in Korea.[6] Kang et al. reported the prevalence, cause of adverse drug events,

Received on March 17, 2015 Revised on April 17, 2015

Accepted on April 22, 2015

Correspondence to: A Jeong Kim, Department of Pharmacy, Seoul National University Hospital, 101 Daehak-ro, Jongno-gu, Seoul 110-744, Korea

Tel: +82-2-2072-3287

E-mail: naanemone@naver.com

*No potential conflict of interest relevant to this article was reported. and types of pharmacist participation in a SICU.[6] Another study demonstrated the prevalence of adverse events and the current status of pharmacist interventions in a pediatric ICU. [7] However, studies examining the causes and types of pharmacist intervention in a medical intensive care unit (MICU) are rare in Korea. 
Previous studies showed that MICU patient were older and had higher severity scores and higher fatality rates than SICU patients. [8] Although, few data directly compare pharmacist interventions in MICUs and SICUs, patients in MICUs need a higher rate of drug information services and total parenteral nutrition consultation than patients in SICUs. $[5,6,9,10]$ These differences influence clinical outcomes of MICU patients.

Therefore, considering the lack of pharmacologic data and aforementioned differences, it is necessary to investigate common drug errors and adverse drug events and pharmacist intervention in a MICU. This study describes the current status and implementation of clinical pharmacy services in a MICU. We evaluated pharmacist interventions and determined the prevalence of interventions and their cause, type, acceptance rate, and relevant medications.

\section{Materials and Methods}

We performed an observational study in the 22-bed MICU of a large tertiary university hospital between September 2013 and August 2014. The medical staff in the ICU consisted of two certified intensivists, one ICU fellow, four residents, registered nurses, two pharmacists, and a dietitian. Our MICU has an open system, which means the physician outside the ICU is responsible for the patient, and the intensivist is a consultant without primary responsibility for any patient. The admission of patients to the ICU is determined by residents and staff on duty based on each patient's medical conditions. All MICU patients 18 years and older who received a pharmacist's intervention were included in the study. No other exclusion criteria were applied.

During the study period, pharmacists performed 1211 pharmacologic recommendations for 340 patients in the MICU (101 cases/month; 536 per 1,000 monitored patientdays). The clinical characteristics of the study subjects are shown in Table 1. Of the 340 patients, 227 (66.8\%) patients were male. The mean age of the patients was 66 years. The most common cause for admission to the ICU was respiratory failure $(n=198,58.2 \%)$, followed by sepsis $(n=63$, $18.5 \%$ ). The majority of patients were admitted from the internal medicine department, specifically the respiratory and hematology-oncology divisions $(n=242,71.2 \%)$.
Table 1. Clinical characteristics of medical intensive care unit patients

\begin{tabular}{lc}
\hline & $\mathrm{N}=340$ \\
\hline Sex (male) & $227(66.8)$ \\
\hline Mean age (years) & $65.7 \pm 15.1$ \\
\hline Height (cm) & $163.1 \pm 10.8$ \\
\hline Weight (kg) & $58.1 \pm 11.4$ \\
\hline Department & \\
\hline Internal medicine & \\
\hline Respiratory & $137(40.3)$ \\
\hline Hematology-oncology & $105(30.9)$ \\
\hline Cardiology & $35(10.3)$ \\
\hline Gastroenterology & $30(8.8)$ \\
\hline Nephrology & $21(6.2)$ \\
\hline Infection & $10(2.9)$ \\
\hline Emergency medicine & $2(0.6)$ \\
\hline Cause for MICU admission & \\
\hline Respiratory failure & $198(58.2)$ \\
\hline Sepsis & $63(18.5)$ \\
\hline Hemodynamic instability & $49(14.4)$ \\
\hline Postoperative care & $10(2.9)$ \\
\hline Monitoring of consciousness & $4(1.2)$ \\
\hline Other & $16(4.7)$ \\
\hline Dala expressed number (porcentage & \\
\hline
\end{tabular}

Data is expressed as number (percentage) or mean \pm standard deviation. N: number; MICU: medical intensive care unit.

Critical care pharmacists with 4 years of critical care experience and one resident pharmacist participated in multidisciplinary rounds three times a week. Prior to attending the medical rounds, pharmacists reviewed individual patient medications independently. The interventions conducted by the pharmacists were recorded in detail using an electronic documentation form. Patient medication orders and pharmacist intervention data were reviewed by pharmacists using electronic medical records (EMR). The reviewed items included patient baseline characteristics (sex, age, height, weight, department, and cause of admission to the MICU), the requestor for intervention (pharmacist, doctor, nurse, or consultant), the method of communication (at medical rounds, via nurses, by phone, by short message service (SMS), by message service in EMR, through consultation papers, or other), the cause of intervention (inappropriate dosage and administration, parenteral/enteral nutrition (PN/ EN), adverse drug reaction, no drug prescribed, inappropri- 
ate drug selection, request for drug information, drug interactions, drug duplication, inappropriate duration, unnecessary drug use, inappropriate laboratory test, inappropriate insurance criteria, or other), the type of intervention (dosage and administration adjustment, PN/EN consultation, provision of drug information, pharmacokinetics advice, suggestion of drug initiation, alternative drug suggestion, suggestion to discontinue drug, laboratory monitoring modification, insurance criteria change, or other), the acceptance rate (complete acceptance, partial acceptance, or no acceptance), the acceptance time (within 24 hours, between 24 and 48 hours, between 48 and 72 hours, or longer than 72 hours), and the drug requiring intervention. The intervention prevalence rate was calculated as the intervention incidence per 1,000 monitored patient-days. We acquired ethical approval for our study from the Research Review Committee of the hospital (IRB No.H-1502-100-650). Data were processed using SPSS software version 21.0 (SPSS Inc., Chicago, IL, USA). Results are presented as number and percentage.

\section{Results}

The vast majority of interventions were initiated by the pharmacist $(\mathrm{n}=1179,97.4 \%)$. The most common method of communication was the pharmacist's announcement at multidisciplinary rounds in the MICU $(n=1160,95.2 \%)$. Other methods of communicating interventions were by phone (n $=31,2.5 \%)$, by SMS $(n=19,1.6 \%)$, via nurses $(n=8,0.7 \%)$, and by EMR message service $(n=1,0.1 \%)$.

The causes for intervention by a pharmacist are shown in Table 2. The most common cause for pharmacist intervention was inappropriate dosage and administration $(n=431$, $34.5 \%$ ), followed by PN/EN problems ( $\mathrm{n}=229,18.3 \%)$, and adverse drug reactions $(\mathrm{n}=148,11.9 \%)$. Of the cases that required intervention for inappropriate dosage and administration, 219 (50.8\%) interventions were for drug overdose, and $151(35.0 \%)$ interventions were for sub-therapeutic underdose. Of the interventions required for drug overdose, intervention due to abnormal kidney function accounted for $150(79.4 \%)$ cases, and 76 of those cases $(50.6 \%)$ required renal replacement therapy (continuous renal replacement therapy [CRRT], $\mathrm{n}=32$ [42.1\%]; hemodialysis [HD], $\mathrm{n}=44$ [57.9\%]). Of the interventions for sub-therapeutic dosage, abnormal kidney function also accounted for the majority of cases $(\mathrm{n}=79,58.1 \%)$. Of those patients, 65 patients $(82.3 \%)$ required renal replacement therapy (CRRT, $\mathrm{n}=57$ [87.7\%]; $\mathrm{HD}, \mathrm{n}=8[12.3 \%])$. Other reasons for pharmacist intervention included absence of necessary drug prescription, inappropriate drug selection, drug interactions, drug duplication, inappropriate duration, unnecessary drug usage, inappropriate laboratory test, and inappropriate insurance criteria.

The acceptance rates for interventions categorized by time course are shown in Table 3. Physicians accepted

Table 2. Causes for intervention by pharmacists

\begin{tabular}{lc}
\hline & Number (\%) \\
\hline Inappropriate dosage and administration & $431(34.5)$ \\
\hline Parenteral/enteral nutrition & $229(18.3)$ \\
\hline Adverse drug reaction & $148(11.9)$ \\
\hline No drug prescription & $89(7.1)$ \\
Inappropriate drug selection & $73(5.8)$ \\
\hline Request for drug information & $68(5.4)$ \\
\hline Drug interactions & $46(3.7)$ \\
Drug duplication & $24(1.9)$ \\
Inappropriate duration & $25(2.0)$ \\
Unnecessary drug usage & $22(1.8)$ \\
Inappropriate laboratory test & $21(1.7)$ \\
Inappropriate insurance criteria & $2(0.2)$ \\
Other & $70(5.6)$ \\
\hline
\end{tabular}

Table 3. Acceptance rate of pharmacologic intervention recommendations

\begin{tabular}{|c|c|c|c|}
\hline & Number (\%) & Time to acceptance & Number (\%) \\
\hline \multirow[t]{2}{*}{ Complete acceptance } & $635(82.3)$ & $\leq 24$ hour & $602(92.8)$ \\
\hline & & 24-47 hour & $28(4.3)$ \\
\hline \multirow[t]{2}{*}{ Partial acceptance } & $14(1.8)$ & 48-72 hour & $13(2.0)$ \\
\hline & & $>72$ hour & $6(0.9)$ \\
\hline No acceptance & $123(15.9)$ & & \\
\hline
\end{tabular}


$649(84.1 \%)$ pharmacist recommendations, completely in 635 cases and in a modified form in 14 cases. Time to acceptance was less than 24 hours for 602 (92.8\%) cases, between 24 and 48 hours for $28(4.3 \%)$ cases, between 48 and 72 hours for $13(2.0 \%)$ cases, and more than 72 hours for 6 $(0.9 \%)$ cases. The acceptance rate could not be confirmed for 439 cases because of patient death or because interventions consisted only of offering drug information. The acceptance rates varied by department. The acceptance rate was highest in infection ( $\mathrm{n}=15$ of $17,88.2 \%$ ), followed by hematology-oncology $(n=161$ of $184,87.5 \%)$, nephrology $(\mathrm{n}=59$ of $68,86.8 \%)$, and gastroenterology $(\mathrm{n}=74$ of 88 , $84.1 \%)$.

The types of pharmacist interventions are shown in Table 4. Drug dosage and administration adjustment were the most common type of intervention recommended $(n=328$, $26.0 \%$ ). The provision of drug information and adjustment of $\mathrm{PN} / \mathrm{EN}$ were both recommended for 228 cases (18.1\%). Other recommendations included pharmacokinetic advice (n $=118,9.3 \%)$, suggestion to discontinue drugs $(n=99,7.8 \%)$, suggestion to initiate drugs $(\mathrm{n}=94,7.4 \%)$, and alternative drug suggestions $(n=89,7.0 \%)$.

The types of pharmacist intervention for each medical problem encountered in the MICU are shown in Table 5. The most frequent intervention for inappropriate dosage and administration was drug dosage adjustment $(n=294$,
$68.4 \%$ ). The most common intervention for adverse drug reactions was the provision of drug information $(n=74$, $50.0 \%$ ), and the most common intervention for inappropriate drug selection was the recommendation of an alternative drug $(n=53,72.6 \%)$. Discontinuing the drug was the most frequently recommended intervention for drug duplication, inappropriate duration, and unnecessary drug usage.

The medications that most often required intervention were anti-infective drugs $(\mathrm{n}=516,42.6 \%)$. PN/EN regimens $(n=256,21.1 \%)$ required more interventions than other drugs (Fig. 1). Vancomycin, colistimethate, and me-

Table 4.Types of pharmacologic intervention recommended by pharmacists*

\begin{tabular}{lc}
\hline & Number (\%) \\
\hline Adjustment of dosage and administration & $328(26.0)$ \\
\hline Adjustment of parenteral/enteral nutrition & $228(18.1)$ \\
\hline Provision of drug information & $228(18.1)$ \\
\hline Pharmacokinetics advice & $118(9.3)$ \\
Suggestion to discontinue drug & $99(7.8)$ \\
Suggestion of drug initiation & $94(7.4)$ \\
Recommendation for alternative drug & $89(7.0)$ \\
Recommendation or modification of laboratory monitoring & $20(1.6)$ \\
Change in insurance criteria & $1(0.1)$ \\
Other & $58(4.6)$ \\
\hline
\end{tabular}

*It is possible to select more than one choice in pharmacologic intervention.

Table 5. Cause of intervention and type of intervention recommended*

\begin{tabular}{lccccccccc}
\hline & Number (\%) & A & B & C & D & E & F & G & H \\
\hline $\begin{array}{l}\text { Inappropriate dosage and admin- } \\
\text { istration }\end{array}$ & $430(35.5)$ & $294(68.4)$ & $0(0.0)$ & $49(11.4)$ & $85(19.8)$ & $6(1.4)$ & $8(1.9)$ & $1(0.2)$ & $9(2.1)$ \\
Parenteral/enteral nutrition & $229(18.3)$ & $0(0.0)$ & $223(97.4)$ & $6(2.6)$ & $0(0.0)$ & $2(0.9)$ & $1(0.4)$ & $0(0.0)$ & $0(0.0)$ \\
\hline $\begin{array}{l}\text { Adverse drug reaction } \\
\text { No drug prescription }\end{array}$ & $148(11.9)$ & $12(8.1)$ & $2(1.4)$ & $74(50.0)$ & $7(4.7)$ & $1(0.7)$ & $16(10.8)$ & $34(23.0)$ & $1(1.4)$ \\
Inappropriate drug selection & $89(7.1)$ & $1(1.1)$ & $1(1.1)$ & $3(3.4)$ & $0(0.0)$ & $80(89.9)$ & $2(2.2)$ & $2(2.2)$ & $0(0.0)$ \\
Drug interactions & $73(5.8)$ & $1(1.4)$ & $0(0.0)$ & $6(8.2)$ & $0(0.0)$ & $4(5.5)$ & $53(72.6)$ & $12(16.4)$ & $0(0.0)$ \\
Drug duplication & $46(3.7)$ & $2(4.3)$ & $0(0.0)$ & $31(67.4)$ & $5(10.9)$ & $0(0.0)$ & $6(13.0)$ & $2(4.3)$ & $0(0.0)$ \\
Inappropriate duration & $24(1.9)$ & $2(8.3)$ & $0(0.0)$ & $0(0.0)$ & $0(0.0)$ & $0(0.0)$ & $1(4.2)$ & $22(91.7)$ & $0(0.0)$ \\
Unnecessary drug usage & $25(2.0)$ & $5(20.0)$ & $0(0.0)$ & $2(8.0)$ & $0(0.0)$ & $0(0.0)$ & $0(0.0)$ & $17(68.0)$ & $2(8.0)$ \\
Inappropriate laboratory test & $22(1.8)$ & $4(18.2)$ & $0(0.0)$ & $0(0.0)$ & $0(0.0)$ & $1(4.5)$ & $0(0.0)$ & $14(63.6)$ & $3(13.6)$ \\
\hline
\end{tabular}

*It is possible to select more than one choice in pharmacologic intervention.

A, adjust dosage and administration; B, Adjust parenteral/enteral nutrition; C, Provision of drug information; D, Pharmacokinetics advice; E, Suggestion to initiate drug; F, Recommend alternative drug; $G$, Suggestion to discontinue drug; $H$, Other. 


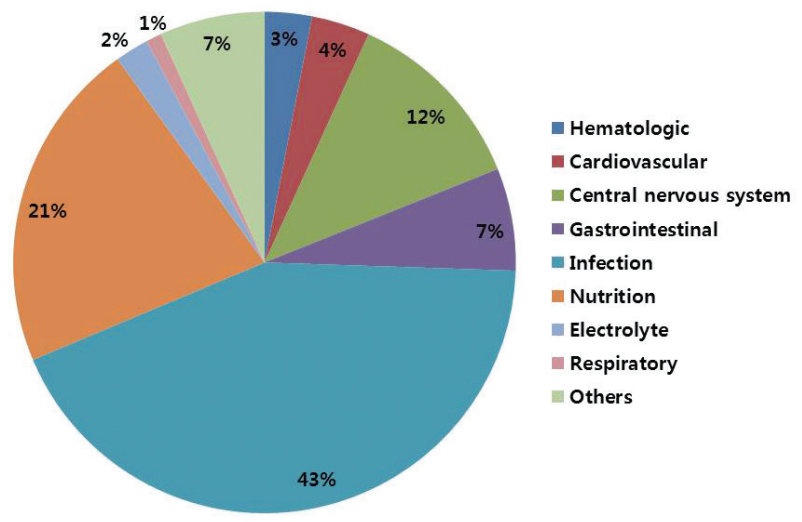

Fig. 1. Drug classification of pharmacist's intervention case.

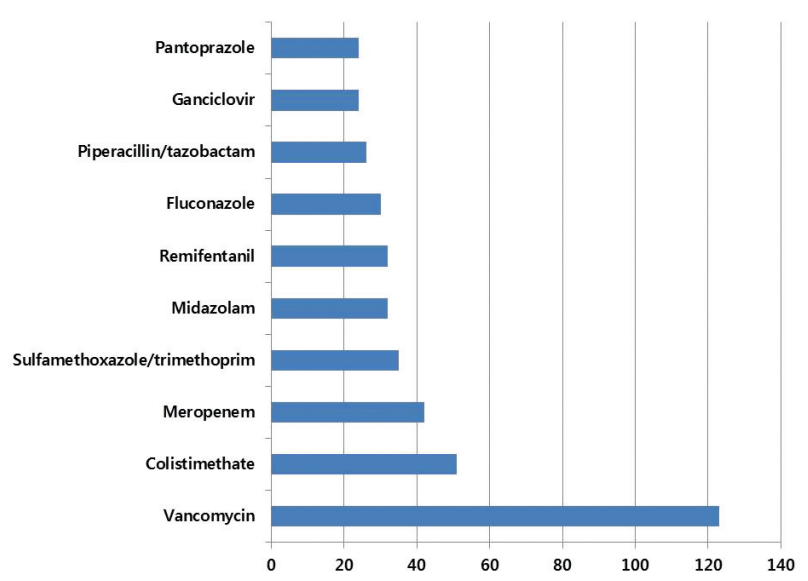

Fig. 2. Common drug which is requiring pharmacist's intervention.

ropenem were the drugs most frequently associated with intervention. The list of drugs associated with pharmacist intervention in more than 20 cases, excluding nutritional supplies, is shown in Fig. 2.

\section{Discussion}

Pharmacist intervention in the ICU is increasing. Multiple drug therapy and the severity of disease among MICU patients result in many adverse drug events and prescription errors. [11,12] It has been shown previously that pharmacist interventions are important in preventing those problems. $[2,6,13,14]$ However, studies examining pharmacotherapeutic problems requiring the intervention of pharmacists in the
MICU are limited in Korea. Thus, we aimed to describe the characteristics of pharmacist interventions in the MICU of a Korean hospital.

In this study, the prevalence of intervention was 536 per 1,000 monitored patient days or an average of 3.3 recommendations per patient evaluated by pharmacists. Actually, the intervention rate among studies is quite different. In previous studies, restricted items mainly associated with patient safety, such as medication errors or adverse drug events, were reported.[2,9,12,15] However, in this study, we described all of the interventions that pharmacy services can offer, including the prevention of medication errors and adverse drug events, adverse drug reactions already encountered, refinements to nutritional regimens, and the provision of drug information. In addition, differences in classification and documentation of interventions might explain the differences in observed intervention incidence rates.

The rate of intervention acceptance in our study group was similar to what has been reported previously.[2,6,15] The acceptance rate was more than $80 \%$, indicating that pharmacist interventions were well accepted by physicians. In addition, more than $90 \%$ of the pharmacist recommendations were accepted within 24 hours, which is higher than what was reported for the SICU.[6] This difference might be caused by the interactive discussion that occurs during multidisciplinary rounds and the rapid reaction of primary physicians. The infection and hematologyoncology departments most actively accepted the recommendations, followed by nephrology, and gastroenterology. The hematology-oncology and infection departments often administer multiple antibiotics and antifungal agents to patients. Therefore, problems such as drug interactions, adverse drug reactions, and the need for drug concentration measurements would have increased the acceptance rate of pharmacist interventions. In the case of nephrology, most patients already have impaired renal function and get renal replacement therapy, which might make the acceptance of pharmacologic recommendations critical for them. Also, considering appropriate nutrition supply for fasting patients in gastroenterology is important, which explains the high acceptance rate in this department.

Most pharmacist intervention requests occurred during multidisciplinary rounds in the MICU, indicating that the pharmacist recommendations were proactive. The pharma- 
cist reviewed the status of patients in advance using electronic documents and communicated with physicians and provided advice at the rounds. Although we did not evaluate the effects of interventions in this study, other studies have shown that proactive recommendations by pharmacists can prevent drug related errors.[16,17]

The three most common causes of intervention in the MICU were inappropriate dosage and administration, PN/ EN problems, and adverse drug reactions, which is similar to what has been reported previously.[2] Those conditions are associated with a high rate of multiple drug prescriptions and impaired organ function. Changes in drug absorption, distribution, metabolism, and excretion caused by multiple organ dysfunction and drug interactions can result in improper drug dosage and adverse drug reactions. Thus, it is important for pharmacists to continuously review medication prescribed to critically ill patients to ensure drug efficacy and patient safety. Of the patients that required intervention for improper drug dosage, $61.8 \%$ had impaired renal function, and $38.1 \%$ received renal replacement therapy. Due to changes in CRRT modes and doses and lack of specific data, it is not easy to optimize medication therapy and minimize under- or over-dosing in those patients. It has been shown previously that dosage adjustment is frequently required for kidney dysfunction.[13,14] In addition, extracorporeal membrane oxygenation also acts as a challenging factor in the optimization of drug dosage. Furthermore, we confirmed that PN/EN problems account for a high proportion of required interventions in our department, although previous studies have not found the same thing.

The most common types of interventions were drug dosage and administration adjustment, refinement of PN/EN regimens, and the provision of drug information. Changes to the clinical status of patients, in particular to pharmacokinetics as a result of organ dysfunction, result in pharmacologic challenges for clinicians. It is interesting that the provision of drug information is one of the most common interventions. Although physicians are well trained in disease detection, they tend to be unfamiliar with the pharmacokinetics or details of prescribed medications. During the interactive multidisciplinary rounds, physicians asked many questions about drug information. Pharmacists provided needed information about pharmacokinetics, medication formulations, and methods of administration. In addition, physicians have difficulty predicting drug interactions or adverse drug reactions that can result from the prescription of multiple drugs. Pharmacist intervention is effective at providing in-service education to the team, as well as providing information about drug activities.

Pharmacists frequently changed antimicrobial drugs, nutritional products, and central-nervous-system-related drugs. It has been shown previously that antibiotics are the drugs most commonly associated with prescription errors.[9,10,18] The high prevalence of infection in MICU patients could explain the high intervention rate. Pharmacists also made recommendations for other items, including cardiovascular therapy and fluid or electrolyte imbalance management. The role pharmacists play in those cases is in the safe use of drugs and the notification of possible adverse drug reactions.[19]

This study has a few limitations. It was conducted at a single center retrospectively. In addition, this study examined only patients receiving recommendations from pharmacists and did not include an evaluation of the clinical benefits of pharmacist recommendations.

In summary, this study reported on pharmacotherapeutic problems and pharmacist interventions in the MICU of a Korean tertiary hospital and confirmed that the interventions are well accepted by medical teams. In the MICU, inappropriate dosage and administration, PN/EN problems, and adverse drug reactions were the most common pharmacologic problems requiring pharmacist intervention. The recommended interventions included drug dosage or administration adjustment, refinement of the PN/EN regimen, and the provision of drug information. Antimicrobial drugs most frequently required intervention.

To our knowledge, this is the first study to investigate pharmacist interventions in a MICU in Korea. This study provides insight into pharmacy services in the MICU system. The prescription of multiple complex medications can result in medication errors or adverse drug effects. Thus, the involvement of pharmacists to rationalize medication therapy in the MICU could improve drug efficacy and patient safety, as well as increase physicians' understanding of drugs. 


\section{References}

1) Montazeri M, Cook DJ: Impact of a clinical pharmacist in a multidisciplinary intensive care unit. Crit Care Med 1994; 22: 1044-8.

2) Klopotowska JE, Kuiper R, van Kan HJ, de Pont AC, Dijkgraaf MG, Lie-A-Huen L, et al: On-ward participation of a hospital pharmacist in a Dutch intensive care unit reduces prescribing errors and related patient harm: an intervention study. Crit Care 2010; 14: R174.

3) Kopp BJ, Mrsan M, Erstad BL, Duby JJ: Cost implications of and potential adverse events prevented by interventions of a critical care pharmacist. Am J Health Syst Pharm 2007; 64: 2483-7.

4) Anthes AM, Harinstein LM, Smithburger PL, Seybert AL, Kane-Gill SL: Improving adverse drug event detection in critically ill patients through intensive care unit transfer summary screening. Pharmacoepidemiol Drug Saf 2013; 22: 510-6.

5) Kim JM, Park SJ, Sohn YM, Lee YM, Yang CS, Gwak HS, et al: Development of clinical pharmacy services for intensive care units in Korea. Springerplus 2014; 3: 34.

6) Kang M, Kim A, Cho Y, Kim H, Lee H, Yu YJ, et al: Effect of clinical pharmacist interventions on prevention of adverse drug events in surgical intensive care unit. Korean J Crit Care Med 2013; 28: 17-24.

7) Oh YK, Lee GS, Rhie S: Neonatal total nutritional pharmacy service at intensive care unit at a university hospital in Korea. Kor J Clin Pharm 2013; 23: 167-74

8) Craven DE, Kunches LM, Lichtenberg DA, Kollisch NR, Barry MA, Heeren TC, et al: Nosocomial infection and fatality in medical and surgical intensive care unit patients. Arch Intern Med 1988; 148: 1161-8.

9) Jiang SP, Chen J, Zhang XG, Lu XY, Zhao QW: Implementation of pharmacists' interventions and assessment of medication errors in an intensive care unit of a Chinese tertiary hospital. Ther ClinRisk Manag 2014; 10:
861-6.

10) Saokaew S, Maphanta S, Thangsomboon P: Impact of pharmacist's interventions on cost of drug therapy in intensive care unit. Pharm Pract (Granada) 2009; 7: 81-7.

11) Moyen E, Camiré E, Stelfox HT: Clinical review: medication errors in critical care. Crit Care 2008; 12: 208.

12) Camiré E, Moyen E, Stelfox HT: Medication errors in critical care: risk factors, prevention and disclosure. CMAJ 2009; 180: 936-43.

13) Cullen DJ, Sweitzer BJ, Bates DW, Burdick E, Edmondson A, Leape LL: Preventable adverse drug events in hospitalized patients: a comparative study of intensive care and general care units. Crit Care Med 1997; 25: 1289-97.

14) Kane-Gill S, Rea RS, Verrico MM, Weber RJ: Adversedrug-event rates for high-cost and high-use drugs in the intensive care unit. Am J Health Syst Pharm 2006; 63: 1876-81.

15) Zaidi ST, Hassan Y, Postma MJ, Ng SH: Impact of pharmacist recommendations on the cost of drug therapy in ICU patients at a Malaysian hospital. Pharm World Sci 2003; 25: 299-302.

16) Kaushal R, Bates DW, Abramson EL, Soukup JR, Goldmann DA: Unit-based clinical pharmacists' prevention of serious medication errors in pediatric inpatients. Am J Health-Syst Pharm 2008; 65: 1254-60.

17) Leape LL, Cullen DJ, Clapp MD, Burdick E, Demonaco HJ, Erickson JI, et al: Pharmacist participation on physician rounds and adverse drug events in the intensive care unit. JAMA 1999; 282: 267-70.

18) Agalu A, Ayele Y, Bedada W, Woldie M: Medication prescribing errors in the intensive care unit of Jimma University Specialized Hospital, Southwest Ethiopia. J Multidiscip Healthc 2011; 4: 377-82.

19) Bourne RS, Choo CL: Pharmacist proactive medication recommendations using electronic documentation in a UK general critical care unit. Int J Clin Pharm 2012; 34: $351-7$. 